\title{
The rolling ball problem on the sphere
}

\section{Laura M. O. Biscolla}

Universidade Paulista

Rua Dr. Bacelar, 1212, CEP 04026-002 São Paulo, Brasil

Universidade São Judas Tadeu

Rua Taquari, 546, CEP 03166-000, São Paulo, Brasil

E-mail address: laurabiscolla@terra.com.br

\section{Jaume Llibre}

Departament de Matemàtiques, Universitat Autònoma de Barcelona 08193 Bellaterra, Barcelona, Catalonia, Spain

E-mail address: jllibre@mat.uab.cat

\section{Waldyr M. Oliva}

CAMGSD, LARSYS, Instituto Superior Técnico, UTL

Av. Rovisco Pais, 1049-0011, Lisbon, Portugal

Departamento de Matemática Aplicada

Instituto de Matemática e Estatística, USP

Rua do Matão, 1010-CEP 05508-900, São Paulo, Brasil

E-mail address: wamoliva@math.ist.utl.pt

Dedicated to Luís Magalhães and Carlos Rocha on the occasion of their 60th birthdays

\begin{abstract}
By a sequence of rolling motions without slipping or twisting along arcs of great circles outside the surface of a sphere of radius $R$, a spherical ball of unit radius has to be transferred from an initial state to an arbitrary final state taking into account the orientation of the ball. Assuming $R>1$ we provide a new and shorter prove of the result of Frenkel and Garcia in [4] that with at most 4 moves we can go from a given initial state to an arbitrary final state. Important cases such as the so called elimination of the spin discrepancy are done with 3 moves only.
\end{abstract}

1991 Mathematics Subject Classification. Primary 58E25, 93B27.

Key words: Control theory, rolling ball problem. 


\section{Introduction and statement of the results}

The rolling movements of a spherical ball $B$ of unit radius over an oriented connected smooth surface $S$ embedded in $\mathbb{R}^{3}$ suggest the consideration of some special kinematic (virtual) motions. A state of the ball is defined to be the pair formed by the point of contact between $B$ and $S$ and a positive orthonormal frame attached to $B$. Assume that the ball $B$ rolls freely on $S$, i.e. $S \cap B$ is the singleton of the contact point. So the set of all the states is identified with the manifold $M=S \times S O(3)$, where $S O(3)$ denotes the group of orthogonal $3 \times 3$ matrices.

A move is a smooth path on $M$ corresponding to a rolling of $B$ on $S$ without slipping or twisting along a geodesic of the surface $S$. No slipping in the rolling means that at each instant the point of contact between $B$ and $S$ has zero velocity; no twisting means that, at each instant, the axis of rotation must be parallel to the tangent plane of $S$ at the point of contact.

According with John M. Hammersley the following problem was proposed by David Kendall in the 1950s: What is the number $N$ of moves necessary and sufficient to reach any final state of $\mathbb{R}^{2} \times S O(3)$ starting at a given initial state? In an interesting paper Hammersley [6] shows that $N=3$. Recently we have provided a new and short proof of this result, see $[3]$.

The following historical considerations we quote from $[6]$ p.112: The original version of the question set (by David Kendall in the 1950s) for 18-year-old schoolboys, invited candidates to investigate how two moves, each of length $\pi$, would change the ball's orientation; and to deduce in the first place that $N \leq 11$, and in the second place that $N \leq 7$. Candidates scored bonus marks for any improvement on 7 moves. When he first set the question, Kendall knew that $N \leq 5$; but, interest being aroused amongst professional mathematicians at Oxford, he and others soon discovered that the answer must be either $N=3$ or $N=4$. But in the 1950 s nobody could decide between these two possibilities. There was renewed interested in the 1970s, and not only amongst professional mathematicians: for example the President of Trinity (a distinguished biochemist) spent some time rolling a ball around his drawing room floor in search of empirical insight. In 1978, while delivering the opening address to the first Australasian Mathematical Convention, I posed the problem to mathematicians down under; but I have not subsequently received a solution from them. So this is an opportunity to publish the solution.

Hammersley [6] p.130 and Jurdjevic [7] are also interested in the same problem but when the spherical ball of unit radius rolls without slipping or twisting through arcs of great circles outside a sphere $\mathbb{S}^{2}$ of radius $R>1$. In 
this case the manifold of states is identified with the set $\mathbb{S}^{2} \times S O(3)$. In the more general case $M=S \times S O(3)$ one asks: What is the minimum number $N(S)$ of moves sufficient to reach any final state of $S \times S O(3)$ starting at a given initial state? For the case $S=\mathbb{S}^{2}$ (the sphere of radius $R$ ), it is known that $N\left(\mathbb{S}^{2}\right)$ is finite if $R \neq 1$, see Jurdjevic [7] pages 165-169 (section 5.1) and Biscolla [2]. In fact, from the results of this last paper it can be proved that the number $N(S)$ is finite when the unit ball rolls without slipping or twisting along geodesics of an analytic oriented connected and compact surface of revolution which has everywhere Gaussian curvature different from 1.

From now on we assume that all rolling motions are done outside the surface of the sphere $\mathbb{S}^{2}$.

Recently, Frenkel and Garcia [4] proved that if $\mathbb{S}^{2}$ is a sphere of radius $R>1$, then $3 \leq N\left(\mathbb{S}^{2}\right) \leq 4$. In Theorem 8 of this paper, it is proved, for any $R>1$ and only three moves, the so called "elimination of the spin discrepancy" (see [6]). We also provide a new and shorter proof of the result of Frenkel and Garcia [4], that if $R>1$, with at most 4 moves we can go from a given initial state to an arbitrary final state (see Theorem $7)$.

We remark that the rolling ball problem on the sphere has special interest in control theory and in robotics, see [7].

The paper is organized as follows. In section 2 we recall the Euler angles and using these angles can get an easy representation of the elements of $S O(3)$. In section 3 we show that four moves are sufficient on $\mathbb{S}^{2}$ with $R>1$. In section 4 we see two important cases in which 3 moves are sufficient on $\mathbb{S}^{2}$ including the elimination of the spin discrepancy .

\section{Euler angles}

According to Euler's rotation theorem, any rotation of $\mathbb{R}^{3}$, i.e. any element of the $S O(3)$, may be described using three angles. If the rotations are written in terms of rotation matrices $R_{3}(a), R_{1}(b)$ and $R_{3}(c)$, then a general rotation $M \in S O(3)$ can be written as

$$
M=R_{3}(c) R_{1}(b) R_{3}(a),
$$

where

$$
R_{3}(a)=\left(\begin{array}{ccc}
\cos a & \sin a & 0 \\
-\sin a & \cos a & 0 \\
0 & 0 & 1
\end{array}\right), \quad R_{1}(b)=\left(\begin{array}{ccc}
1 & 0 & 0 \\
0 & \cos b & \sin b \\
0 & -\sin b & \cos b
\end{array}\right)
$$


The three angles $a, b$ and $c$ are called Euler angles. There are several conventions for Euler angles, depending on the axes about which the rotations are carried out. The so-called $x$-convention is the one we use, being also the most common definition. In this convention a rotation is given by Euler angles $(a, b, c)$ where the first rotation is by an angle $a$ about the $z$-axis, the second is by an angle $b$ in $[0, \pi]$ about the $x$-axis and the third is by an angle $c$ about the $z$-axis.

The Euler angles are also related to the quaternions, which was the approach used by Hammersley to study the rolling ball ball problem in the plane (see [6]). For more details about the Euler angles see for instance [5].

A state $(P, M) \in \mathbb{S}^{2} \times S O(3)$ of the spherical ball on the surface of the sphere $\mathbb{S}^{2}$ means that the contact point of the ball with the sphere is the point $P$ of $\mathbb{S}^{2}$ and its orientation is given by the rotation matrix $M$ or its associated orthonormal frame $(I, J, K)$ of $\mathbb{R}^{3}$, where the vectors $I, J$ and $K$ are the first, second and third column of the matrix $M$, respectively. Moreover the state $(P, M)$ is also denoted by $(P,(I, J, K))$.

We denote by $(i, j, k)$ the orthonormal frame $(I, J, K)$ associated to the $3 \times 3$ identity matrix $I d$.

\section{Four moves are sufficient on $\mathbb{S}^{2}$}

Let $\mathbb{S}^{2}=\left\{(x, y, z) \in \mathbb{R}^{3}: x^{2}+y^{2}+z^{2}=R^{2}\right\}$. The next proposition and their corollaries essentially follow [4].

Proposition 1. Let $\{i, j, k\}$ be the frame attached to the unitary ball of center $C=(0,0, R+1)$ with the vector $k$ orthogonal to the sphere $\mathbb{S}^{2}$ of radius $R$ going from $C$ to $A=(0,0, R+2)$. Assume that the ball rolls along the meridian $\mathcal{M}$ of $\mathbb{S}^{2}$ defined by the plane $j-k$. If after a rolling the vector $k$ goes to a vector $u$ making an angle $\alpha$ with $k$, then the distance $s$ (along the meridian $\mathcal{M}$ ) is equal to $s=R \alpha /(R+1)$, and the point $A$ goes to a point $\bar{A}$, such that $u$ is the vector from $\bar{C}$ (the new position of the center of the ball) to $\bar{A}$.

Proof. It is easy to check that $\alpha=s+s / R$. So we get that $s=R \alpha /(R+1)$.

Given two points $P$ and $Q$ on $\mathbb{S}^{2}$ the distance between $P$ and $Q, d(P, Q)$, is the length of the small arc of the great circle containing $P$ and $Q$. In particular if $P$ and $Q$ are two points on $\mathbb{S}^{2}$ whose angle between them is $2 \pi /(R+1)$, the distance $d(P, Q)$ is equal to $2 \pi R /(R+1)$. 
When rolling a ball from an initial state $(P, M)$ in $\mathbb{S}^{2} \times S O(3)$ to a final state $(Q, M)$ it is considered that $P$ goes to $Q$ along the arc of small length of the great circle containing $P$ and $Q$.

Hereafter when we say that a distance between two points is larger than $2 \pi n R$ and smaller than $2 \pi(n+1) R$ we mean that we have done more than $n$ and less than $n+1$ turns on the sphere along the great circle. With this definition we have that $d(P, P)=2 \pi n R$ with $n=0,1,2, \ldots$

Corollary 2. If we start rolling the ball at the north pole of the sphere $\mathbb{S}^{2}$ with the orientation $M \in S O(3)$, then the curves on $\mathbb{S}^{2}$ on which the orientation of the ball is $M$ are parallels at a distance $2 \pi n R /(R+1)$ of the north pole for $n=1,2, \ldots$ It can happen that some of these parallels can be reduced to the north or south pole (if $R+1$ divides $2 n$ ).

Proof. When the ball starting to roll at the north pole reaches in one move a point of the parallel whose distance to the north pole is $2 \pi n R /(R+1)$, the ball has done exactly $n$ complete turns. Therefore, the corollary follows.

Corollary 3. Rolling the ball from the initial state $(P,\{i, j, k\})$ along a great circle of length $\pi R /(R+1)+2 \pi n R /(R+1)$, with $n$ a positive integer, then the orientation of the ball at the final state is given by an orthonormal frame of the form $\{I, J,-k\}$.

Lemma 4. Let $\gamma$ and $\sigma$ be two great circles of $\mathbb{S}^{2}$, and let $P_{0}$ and $Q_{0}$ be two arbitrary points on $\gamma$ and $\sigma$ respectively. Consider $S_{\gamma}\left(\right.$ resp. $\left.S_{\sigma}\right)$ to be the set of points $P_{n}$ of $\gamma($ resp. of $\sigma)$ which are at distance $2 \pi n R /(R+1)$ of the point $P_{0}$ for $n=1,2, \ldots$ Then the distance between the points of $S_{\gamma}$ and $S_{\sigma}$ is smaller than $2 \pi R /(R+1)$.

Proof. If $\gamma$ coincides with $\sigma$ the proof is trivial. Assume that $\gamma$ is different from $\sigma$. Let $A$ be one of the two points of the intersection between $\gamma$ and $\sigma$. Clearly there are points of $S_{\gamma}$ whose distance to $A$ is at most $\pi R /(R+1)$ and let $P_{r}$ be one of such points. Similarly there is a point $Q_{s}$ of $S_{\sigma}$ whose distance to $A$ is at most $\pi R /(R+1)$. Then the distance between $P_{r}$ and $Q_{s}$ is smaller than $2 \pi R /(R+1)$.

Lemma 5. Let $R>1, M \in S O(3)$ and $P, Q \in \mathbb{S}^{2}$ with $P \neq Q$. If the distance between $P$ and $Q$ is smaller than $2 \pi R /(R+1)$ we can go from the initial state $(P, M)$ to the final state $(Q, M)$ with 2 moves; otherwise we need 3 moves.

Proof. Without loss of generality we can take $P$ as the north pole of $\mathbb{S}^{2}$. By Corollary 2 and Lemma 4 it is clear that with one move we may assume that on the sphere the distance $d(P, Q)$ between $P$ and $Q$ is less than or 
equal to $2 \pi R /(R+1)$. We claim that given two different points $P$ and $Q$ we have that

$$
d(P, Q) \leq \frac{2 \pi R}{R+1} \quad \text { implies } \quad C_{P} \cap C_{Q} \neq \emptyset
$$

where $C_{P}=\bigcup_{n=1}^{\infty} C P_{n}$ with

$$
C P_{n}=\left\{X \in \mathbb{S}^{2}: d(X, P)=n \frac{2 \pi R}{R+1}\right\} .
$$

Now we consider the following two cases.

Case 1: $R$ is irrational. Then the set of parallels $C_{P}$ and $C_{Q}$ are dense in $\mathbb{S}^{2}$, and since $P \neq Q$ they intersect. So the claim is proved in this case.

Case 2: $R$ is rational. Then the set of parallels $C_{P}$ and $C_{Q}$ is finite. Let $\gamma$ be the great circle passing through $P$ and $Q$. The intersection of $\gamma$ with $C_{P}$ is a finite set of equidistant points on $\gamma$ which are located symmetrically with respect to the diameter of $\gamma$ passing through $P$. If some of the points of $\gamma \cap C_{P}$ different from $P$ are located in the closed north hemisphere of $\mathbb{S}^{2}$, it is clear that $C Q_{1} \cap C P_{1} \neq \emptyset$. Otherwise we must have either $2 \pi R /(R+1)=\pi R$, or $2 \pi R /(R+1)=2 \pi R / 3$. In the first case $R=1$, which contradicts the hypothesis that $R>1$.

Assume $2 \pi R /(R+1)=2 \pi R / 3$, then $R=2$ and $C_{P}$ is formed by the point $P$ and the parallel which is at a distance $2 \pi R / 3$ of $P$. Again it follows easily that $C Q_{1} \cap C P_{1} \neq \emptyset$. Hence the claim is proved.

So by Corollary 2 when $d(P, Q) \leq 2 \pi R /(R+1)$, it follows that 2 moves are sufficient to go from $(P, M)$ to $(Q, M)$; it is enough to go from $(P, M)$ to $(Z, M)$ where $Z$ is a point of $C_{P} \cap C_{Q}$ and from $(Z, M)$ to $(Q, M)$.

It is well known that the rotation matrix $\mathcal{R}\{e, \delta\}$ of angle $\delta$ around an axis through the origin with direction $e=(u, v, w)$ is

$$
\frac{1}{L^{2}}\left(\begin{array}{ccc}
u^{2}+\left(v^{2}+w^{2}\right) \cos \delta & u v(1-\cos \delta)-w L \sin \delta & u w(1-\cos \delta)+v L \sin \delta \\
u v(1-\cos \delta)+w L \sin \delta & v^{2}+\left(u^{2}+w^{2}\right) \cos \delta & v w(1-\cos \delta)-u L \sin \delta \\
u w(1-\cos \delta)-v L \sin \delta & v w(1-\cos \delta)+u L \sin \delta & w^{2}+\left(u^{2}+v^{2}\right) \cos \delta
\end{array}\right)
$$

where $L=\sqrt{u^{2}+v^{2}+w^{2}}$.

Now we shall describe the equation of any move starting at the north pole $(0,0, R)$ of $\mathbb{S}^{2}$. The great circles through the north pole are called meridians. Let $\mathcal{M}$ be the meridian contained in the plane through the origin with director vector $e=(\cos \alpha,-\sin \alpha, 0)$. Then, by Proposition 1 , the move of length $l$ starting at the north pole through the meridian $\mathcal{M}$ is 
given by the matrix

$$
\mathcal{R}\{e, \delta\} \quad \text { with } \delta=l(R+1) / R .
$$

More precisely if we have at the north pole the ball with the orientation given by the matrix $M \in S O(3)$, after the move $\mathcal{R}\{e, \delta\}$ the ball has the orientation $\mathcal{R}\{e, \delta\} M$.

Lemma 6. The following two statements hold.

(a) Starting at the north pole with the orientation $\{i, j, k\}$, for every $n \in \mathbb{Z}$ we can pass with one move to points $P^{n}$ of $\mathbb{S}^{2}$ with the orientation given by the matrix

$$
\left(\begin{array}{ccc}
\cos (a-c) & -\sin (a-c) & 0 \\
-\sin (a-c) & -\cos (a-c) & 0 \\
0 & 0 & -1
\end{array}\right),
$$

where $a$ and $c$ are arbitrary angles of $\mathbb{S}^{1}$. The distance between the points $P^{n}$ and $P^{n+1}$ is $2 \pi R /(R+1)$.

(b) Starting at the north pole with an arbitrary orientation given by the matrix $R_{3}(c) R_{1}(b) R_{3}(a)$ with $a, c \in \mathbb{S}^{1}$ and $b \in[0, \pi]$ (see section $2)$, for every $n \in \mathbb{Z}$ we can pass with one move to points $Q^{n}$ of $\mathbb{S}^{2}$ with the orientation given by the matrix (2). The distance between the points $Q^{n}$ and $Q^{n+1}$ is $2 \pi R /(R+1)$.

Proof. For every $n \in \mathbb{Z}$ take the move $\mathcal{R}\{e, \delta\} I d$ given by (1) of length $l=\pi R /(R+1)+n 2 \pi R /(R+1)$ through the meridian $\mathcal{M}$ with director vector $e$ having $\alpha=(c-a) / 2$.

The point $P^{n}$ is the image of the north pole $(0,0, R)$ under the matrix $\mathcal{R}\{e, \delta\}$. This completes the proof of statement (a).

For every $n \in \mathbb{Z}$ take the move $\mathcal{R}\{e, \delta\} R_{3}(c) R_{1}(b) R_{3}(a)$ through the meridian $\mathcal{M}$ with director vector $e$ having $\alpha=c$, where $\mathcal{R}\{e, \delta\}$ is given by (1) with length $l=(b+\pi) R /(R+1)+n 2 \pi R /(R+1)$.

The point $Q^{n}$ is the image of the north pole $(0,0, R)$ under the matrix $\mathcal{R}\{e, \delta\}$. This proves statement (b).

We now show that with 4 moves one can go from the state $\left(P_{0},(i, j, k)\right)$ to any other state $\left(P_{1},(I, J, K)\right)$.

Theorem 7. Let $R>1$. Given two points $P, Q \in \mathbb{S}^{2}$ and $M \in S O(3)$, we can pass from the initial state $(P, I d)$ to the final state $(Q, M)$ in 4 moves.

Proof. Let $M=R_{3}(c) R_{1}(b) R_{3}(a)$ with $a, c \in \mathbb{S}^{1}$ and $b \in[0, \pi]$.

Without loss of generality we can assume that the point $P$ is the north pole of the sphere. Then, for every $n \in \mathbb{Z}$, using Lemma 6(a) and doing 
one move we can pass from the state $(P, I d)$ to the states $\left(P^{n}, M_{0}\right)$ where $M_{0}$ is the matrix (2), and where $a, c$ are the angles appearing in the matrix $M$.

Clearly the result proved in Lemma 6(b) can be applied to any point of the sphere, not necessarily the north pole. Therefore, for every $m \in \mathbb{Z}$ using Lemma $6(\mathrm{~b})$ and doing one move we can pass from the state $(Q, M)$ to the states $\left(Q^{m}, M_{0}\right)$ where $M_{0}$ is the matrix (2).

Now by Lemma 4 there are two points $P^{n}$ and $Q^{m}$ whose distance is smaller than $2 \pi R /(R+1)$. Finally, using Lemma 5 , we can pass with two moves from the state $\left(P^{n}, M_{0}\right)$ to the state $\left(Q^{m}, M_{0}\right)$, which concludes the proof.

\section{Cases in which three moves are sufficient on $\mathbb{S}^{2}$}

The next result allows to go with 3 moves from the state $\left(P_{0},(i, j, k)\right)$ to an arbitrary state of the form $\left(P_{0},(I, J, k)\right)$ which corresponds to $\left(P_{0}, R_{3}(\beta)\right)$ for a certain $\beta$; this result is known as the elimination of the spin discrepancy $\beta$.

Theorem 8. Given the state $\left(P_{0},(i, j, k)\right)$ we can pass to the state $\left(P_{0},(I, J, k)\right)$ doing 3 moves.

Proof. As usual we assume that $P_{0}$ is the north pole of the sphere of radius $R$. We consider the spherical equilateral triangle whose vertices are $P_{0}=R(0,0,1)=R p_{0}, P_{1}=R(0, \sin (l / R), \cos (l / R))=R p_{1}$ and $P_{2}=$ $R(-\sin \alpha \sin (l / R), \cos \alpha \sin (l / R), \cos (l / R))=R p_{2}$ and length $l$, where $\alpha$ is the angle between the two great circles having vertex at $P_{0}$. Some basic spherical geometry shows that $\cos \alpha=\cos (l / R)(1-\cos (l / R)) / \sin ^{2}(l / R)$.

Taking into account Proposition 1 the move from the state $\left(P_{0}, I d\right)$ to the state $\left(P_{1}, M_{1}\right)$ through the arc of the great circle of length $l$ going from $P_{0}$ to $P_{1}$ is given by the matrix $M_{1}=\mathcal{R}\left(p_{0} \wedge p_{1} /\left|p_{0} \wedge p_{1}\right|,(1+R) l / R\right)$. Similarly the move from the state $\left(P_{1}, M_{1}\right)$ to the state $\left(P_{2}, M_{2} M_{1}\right)$ through the arc of the great circle of length $l$ going from $P_{1}$ to $P_{2}$ is given by the matrix $M_{2}=\mathcal{R}\left(p_{1} \wedge p_{2} /\left|p_{1} \wedge p_{2}\right|,(1+R) l / R\right)$.

The move from the state $\left(P_{0}, R_{3}(\beta)\right)$ to the state $\left(P_{2}, M_{3} R_{3}(\beta)\right)$ through the arc of the great circle of length $l$ going from $P_{0}$ to $P_{2}$ is given by the matrix $M_{3}=\mathcal{R}\left(p_{0} \wedge p_{2} /\left|p_{0} \wedge p_{2}\right|,(1+R) l / R\right)$. 
It is now enough to prove that there exists $l$ satisfying the equality $M_{2} M_{1}=M_{3} R_{3}(\beta)$. Take $s=\sin (l / R)$ satisfying

$$
\cos \beta=\frac{\sqrt{1-s^{2}}\left(-s^{6}+30 s^{4}-64 s^{2}+32\right)-2\left(5 s^{6}-29 s^{4}+40 s^{2}-16\right)}{s^{6}\left(\sqrt{1-s^{2}}+1\right)} .
$$

When $s$ runs the interval $[0.866025 . ., 1]$ the right hand side of the previous expression covers the interval $[-1,1]$. Hence for every $\beta$ there exists some $l_{0} \in(0, \pi / 2]$ satisfying the above equality. We checked using the symbolic computational system Mathematica that for such a $l_{0}$ we have $M_{2} M_{1}=$ $M_{3} R_{3}(\beta)$.

The next result allows to go with 3 moves from the state $\left(P_{0},(i, j, k)\right)$ to an arbitrary state of the form $\left(P_{1},(I, J,-k)\right)$.

Proposition 9. Let $R>1$. Then, given the state $\left(P_{0},(i, j, k)\right)$, we can pass to the state $\left(P_{1},(I, J,-k)\right)$ doing 3 moves.

Proof. For every $n \in \mathbb{Z}$ using Lemma 6(b) and doing one move we can pass from the state $\left(P_{0},(i, j, k)\right)$ to the states $\left(P^{n},(I, J,-k)\right)$. Since $R>1$, then by Lemma 5 we can pass from some state $\left(P^{n},(I, J,-k)\right)$ to the state $\left(P_{1},(I, J,-k)\right)$ with two moves. Hence the proposition is proved.

\section{Acknowledgments}

We thank to the referee all his comments and suggestions that allow us to improve the presentation and exposition of this paper.

The second author has been supported by the grants MINECO/FEDERSpain MTM2008-03437, CIRIT-Catalonia 2009SGR 410, ICREA Academia and FP7-PEOPLE-2012-IRSES-316338 and 318999. The third author thanks to FCT (Portugal) for the partial support through Program POCTI/FEDER and PDCT/MAT/56476/2004; and thanks also M.V.P. Garcia for many valuable discussions on the subject.

\section{References}

[1] V.I. Arnold And A. Avez, Ergodic problems of classical mechanics, Translated from the French by A. Avez, W. A. Benjamin, Inc., New York-Amsterdam, 1968.

[2] L.M.O. Biscolla, Controlabilidade do rolamento de una esfera sobre una superfície de revoluçao, Ph. D., IME, USP, São Paulo, Brasil, 2005.

[3] L.M.O. Biscolla, J. Llibre AND W.M. Oliva, The rolling ball problem on the plane revisited, to appear in Zeitschrift fuer Angewandte Mathematik und Physik, 2013.

[4] L.M. Frenkel And M.V.P. Garcia, Kendall's problem on a sphere, Qualitative Theory of Dynamical Systems 10 (2011), 333-341. 
[5] H. Goldstein, The Euler Angles and Euler Angles in Alternate Conventions, Section 4.4 and Appendix B in Classical Mechanics, 2nd ed. Reading, MA, Addison-Wesley, pp. 143-148 and 606-610, 1980.

[6] J.M. Hammersley, Oxford commemoration ball, Probability, statistics and analysis, Papers dedicated to David G. Kendall on the occasion of his sixty-fifth birthday. Edited by J. F. C. Kingman and G. E. H. Reuter, London Math. Soc. Lecture Note Ser. 79, Cambridge Univ. Press, Cambridge-New York, pp 112-142, 1983.

[7] V. Jurdjevic, Geometric Control Theory, Cambridge Univ. Press, 1997. 